\title{
Adaptive Fingerprint Binarization by Frequency Domain Analysis
}

\author{
Josef Ström Bartůněk, Mikael Nilsson, Jörgen Nordberg, and Ingvar Claesson \\ Department of Signal Processing, School of Engineering, Blekinge Institute of Technology \\ Box 520, SE-372 25 Ronneby, Sweden \\ E-mails: jsb@bth.se, mkn@bth.se,jno@bth.se, icl@bth.se
}

\begin{abstract}
This paper presents a new approach for fingerprint enhancement by using directional filters and binarization. A straightforward method for automatically tuning the size of local area is obtained by analyzing entire fingerprint image in the frequency domain. Hence, the algorithm will adjust adaptively to the local area of the fingerprint image, independent on the characteristics of the fingerprint sensor or the physical appearance of the fingerprints. Frequency analysis is carried out in the local areas to design directional filters. Experimental results are presented.
\end{abstract}

\section{INTRODUCTION}

Fingertip epidermis depicted on smooth surfaces results in line patterns denoted as fingerprints. The raw fingerprints are usually processed to compensate for factors such as skin condition and sensor noise. Many fingerprint recognition systems use enhancement and separation of fingerprints in a pre-processing step denoted binarization [1]. An interesting binarization category widely used in many algorithms is denoted contextual filtering. The basic concept of the contextual filtering is to design directional filters with their shape aligned to the ridge orientation in each local area of the fingerprint. Among the first papers describing contextual filtering are [2], [3] where a filter mask with horizontal direction is designed based on four parameters that are manually identified from each fingerprint. Additional directions are created by rotating this horizontal filter mask. The method in [4] uses a Butterworth type of bandpass directed filter and the filtering is performed in the frequency domain. Another method in [5] uses a Gabor filter to design the directional filters where also the spatial frequency of each ridge was taken in consideration. A recent technique presented in [6] exploits second directional derivatives to construct these filters, and a method for selecting suitable size of the local area is also proposed.

Various parameters are fixed in the algorithms stated above; some methods have fixed size on the local area while other have fixed filter size. Hence, each algorithm has to be tuned manually to achieve better performance for different fingerprint images. The aim of the method proposed in this paper is to develop an entirely automated fingerprint binarization algorithm. A new approach will be described where fingerprint regions are analyzed in the frequency domain. A technique is derived where the proper size of the local area is automatically determined for each individual fingerprint. Frequency analysis is also carried out in local areas to design directional filters.

\section{Fingerprint BinARIZATION Algorithm}

\section{A. Spatial and Frequency Domains}

The Fast Fourier Transform (FFT) is a method used to map signals from the spatial domain into the frequency domain. An important part of frequency domain analysis involves the amplitude spectrum [7]. In the amplitude spectrum vital characteristics of the signal can be observed. The function

$$
f\left(n_{1}, n_{2}\right)=\cos \left(\frac{2 \pi}{N} k_{1} n_{1}+\frac{2 \pi}{N} k_{2} n_{2}\right)
$$

is used to create a 2D sinusoidal signal, where the constants $N, k_{1}$, and $k_{2}$ are related to the periodicity (frequency) of the sinusoidal signal and $n_{1}$ and $n_{2}$ are the spatial coordinates, see Fig. 1 a) and c). Two sinusoidal signals and corresponding amplitude spectrums $F\left(K_{1}, K_{2}\right)$ are illustrated in Fig. 1 b) and d). It is interesting to observe that the coordinates $\left(K_{1}, K_{2}\right)$ of the dominant peak $P_{D}$ in the amplitude spectrum are the same

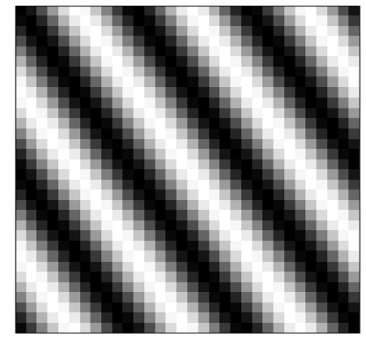

(a)

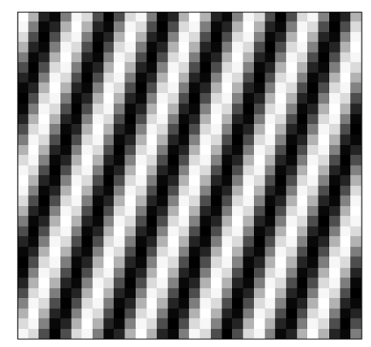

(c)

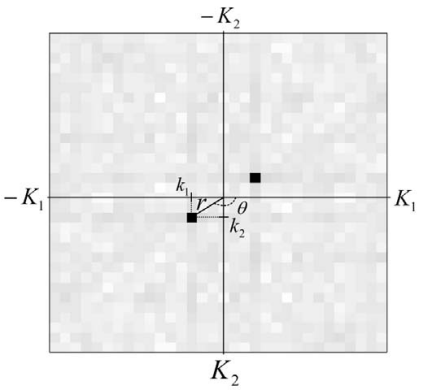

(b)

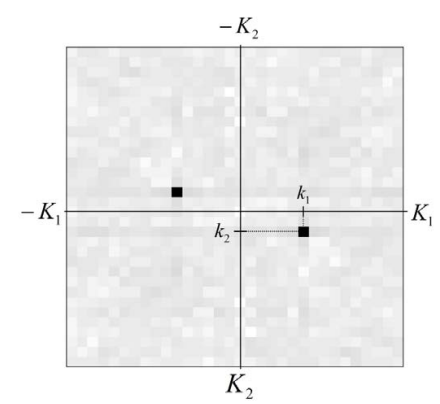

(d)
Fig. 1. Two sinusoidal signals represented in the spatial domain a) and c) and with corresponding amplitude spectrums in b) and d). 
as the parameters $k_{1}$ and $k_{2}$ in (1). An attractive property of the amplitude spectrum is that the distance $r$ from the origin and the angle $\theta$ of the dominant peak $P_{D}$ is proportional to the spatial frequency and the orientation of the sinusoidal signal.

\section{B. Determining the Size of the Local Area}

A circular pattern can be observed around the origin by analyzing the amplitude spectrum of entire fingerprint image, see Fig. 2 a) and b). The observed circular pattern stems from the line pattern in the fingerprint which is oriented in all directions. The dominant spatial frequency in the fingerprint image can be found by estimating the radius of these circular structures. The upper half plane and the lower half plane of the amplitude spectrum are symmetric. Calculations are therefore performed in only one of the regions, the lower half plane.

A straightforward method to find the dominant spatial frequency is performed by computing the distance $r$ from the origin to each point in the amplitude spectrum

$$
r=\sqrt{\omega_{1}^{2}+\omega_{2}^{2}}
$$

where

$$
\omega_{1}=\frac{2 \pi}{N_{1}} k_{1}
$$

and

$$
\omega_{2}=\frac{2 \pi}{N_{2}} k_{2}
$$

All points associated with a certain radius are now summed and presented in the upper graph of Fig. $2 \mathrm{c}$ ). The number of points in the amplitude spectrum that are associated to a certain radius $r$ are illustrated in the middle graph of Fig. 2 c). The average value of points associated to a certain radius $r$ are illustrated in the lower part of Fig. 2 c), this corresponds to

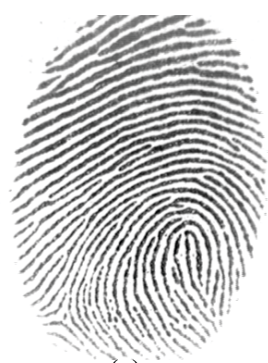

(a)

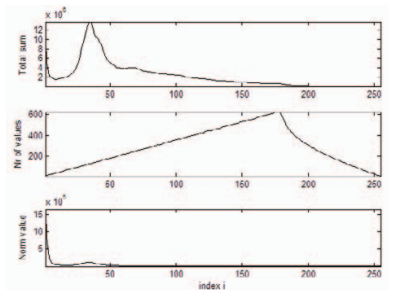

(c)

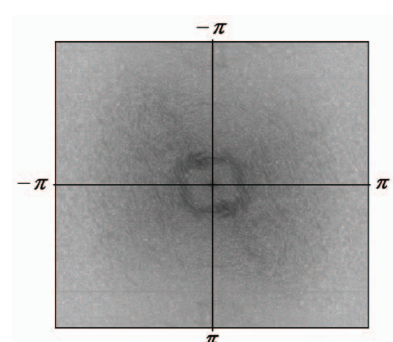

(b)

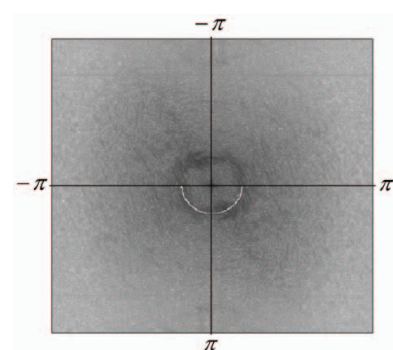

(d)
Fig. 2. a) gray-scale fingerprint image and b) is its amplitude spectrum. c) intermediate graphs used for the semi circle estimation; d) shows the estimated semicircle. the upper graph normalized by the middle graph. The peak value of the lower graph has a radius that corresponds to the dominant spatial frequency of the entire fingerprint image (note that the radius corresponding the very low frequencies are discarded). The semi circle, with a radius corresponding to the dominant spatial frequency, is shown in Fig. $2 \mathrm{~d}$ ).

The frequency bins indices corresponding to the dominant spatial frequencies are found through

$$
k_{1}=\frac{\omega_{f} N_{1}}{2 \pi}
$$

and

$$
k_{2}=\frac{\omega_{f} N_{2}}{2 \pi}
$$

where $\omega_{f}$ is the dominant frequency found through the procedure above.

The spatial frequency represented in pixels is then calculated by

$$
x=\frac{a}{k_{1}}
$$

and

$$
y=\frac{b}{k_{2}}
$$

where $a$ and $b$ denotes the size of the fingerprint image. Then the mean of $x$ and $y$ is calculated and the nearest integers are chosen as the dominant spatial frequency $\Omega_{f}$. The size of local area is selected a multiple of $\Omega_{f}$.

\section{Matched Filter Design}

By comparing Fig. 3 with Fig. 1 many similarities can be observed. The dominant peak $P_{D}$ in Fig. 3 b) is smeared because the signal (image) in Fig. 3 a) is not an ideal sinusoidal signal as in Fig. 1 a). Inserting the coordinates of the $P_{D}$ from the amplitude spectrum in Fig. 3 b) in (1), the original signal in Fig. 3 a) is mimicked by the periodic and even signal in Fig. 1 a). Notice that the orientation and the spatial frequency in the local area are represented with $N, k_{1}$, and $k_{2}$ in the amplitude spectrum, where $N$ is the size of the FFT used and the $k_{1}$ and $k_{2}$ are the coordinates of the $P_{D}$, which may be found by the procedure outlined in section II.B.

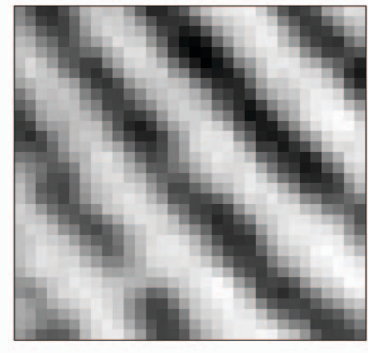

(a)

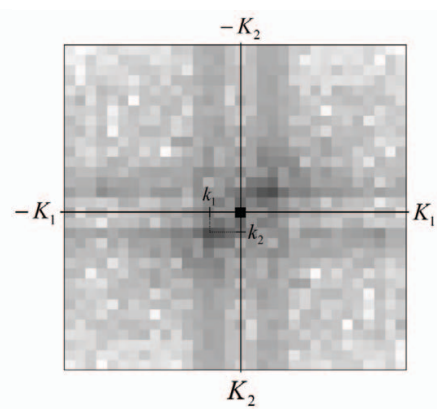

(b)
Fig. 3. a) Local area inside the fingerprint; b) the corresponding amplitude spectrum. 
The mutched filter is obtained by choosing $n_{1}$ and $n_{2}$ such that only one period is selected. The size $S \times S$ of this matched filter is deliberately square and of an odd number so there is a single pixel in the center. The $S$ is calculated as following

$$
S=2 n+1
$$

where $n$ is

$$
n=\operatorname{round}\left(\frac{\pi}{\Delta \omega\left(k_{2}+\left|k_{1}\right|\right)}\right)
$$

and $\Delta \omega$ is

$$
\Delta \omega=\frac{2 \pi}{N} .
$$

The spatial coordinates are chosen as $-n \leq n_{1} \leq n$ and $-n \leq n_{2} \leq n$. The matched filter is tapered by a cosine function

$$
f_{2}\left(n_{1}, n_{2}\right)=\cos \left(\left(\frac{2 \pi}{N} k_{2} n_{1}-\frac{2 \pi}{N} k_{1} n_{2}\right) C\right)
$$

which is obtained through changing the sign and the $k_{1}$ and $k_{2}$ are exchanged in (1) producing $90^{\circ}$ rotation and adding a constant $C$ to control the expansion of the cosine wave. The constant $C$ is calculated as follows

$$
C=\frac{\arccos \left(\frac{0.25}{\cos \left(\Delta \omega k_{1} k n_{1}+\Delta \omega k_{2} k n_{2}\right)}\right)}{\Delta \omega\left(k_{1} k n_{2}-k_{2} k n_{1}\right)}
$$

where $\left(k n_{1}, k n_{2}\right)$ are coordinates of the point on the filter boundary where the top of the cosine is located. The 0.25 denotes that the top on $\left(k n_{1}, k n_{2}\right)$ is attenuated to factor 0.25 of its original value. The final matched directional filter is then created by

$$
g\left(n_{1}, n_{2}\right)=f\left(n_{1}, n_{2}\right) f_{2}\left(n_{1}, n_{2}\right) .
$$

The mean of the filter is subtracted in order to obtain a zero value at the DC component.

\section{Binarization Process}

First the size of the local area is determined. Then the range in each local area are rescaled to range of $[0,255]$. The window is shifted pixel-by-pixel throughout the fingerprint and the coordinates $\left(k_{1}, k_{2}\right)$ of $P_{D}$ are extracted from each amplitude spectrum. The height of $P_{D}$ is used as an indicator if further investigation is necessary. The height of the ideal peak is calculated as

$$
P_{\text {ideal }}=128 \frac{N^{2}}{2}
$$

where the $\mathrm{N}$ is the size of FFT. The ratio between the height of the located peak $P_{D}$ and the height of the ideal peak is calculated as

$$
R=\frac{P_{D}}{P_{\text {ideal }}} .
$$

This ratio of peak heights is illustrated for a typical fingerprint in Fig. 4. A threshold is set from which a decision is made whether or not an extra investigation, in form of zooming in or out of the local area, is necessary. If a better value on $P_{D}$ is found the new $\left(k_{1}, k_{2}\right)$ are extracted. When all coordinates have been extracted a smoothing in the polar

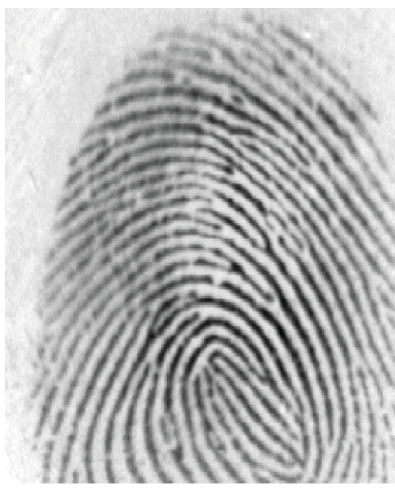

(a)

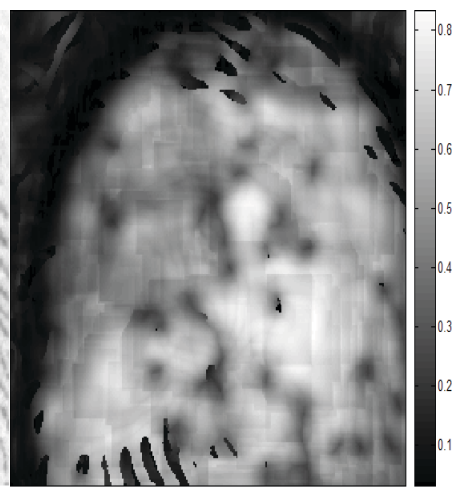

(b)
Fig. 4. a) original fingerprint b) analysis of the periodicity in the a).

coordinate domain is performed by combining the mean value and a median filtering [7]. Then the filters are created from the smoothed coordinates according to section II.C and filtering is carried out in the spatial domain. Binarization is following directly after the filtering stage by thresholding. The center region $p$ in each local area with the same size as the filter is acquired and processed according to

$$
z=\sum_{n_{1}=-n}^{n} \sum_{n_{2}=-n}^{n} p\left(n_{1}, n_{2}\right) g\left(n_{1}, n_{2}\right)
$$

If $z>0$ the pixel is 1 (white) othervise it is 0 (black). After binarization a postprocessing is performed by median filtering the binary fingerprint image.

Determination of fingerprint mask is based on peaks ration. The regions with enough high ratios are considered as part of the fingerprint.

\section{EXPERIMENTAL RESULTS}

The proposed algorithm was tested on numerous fingerprint images taken from the different databases found in [1]. All fingerprints have been obtained from different sensors and therefore have varying characteristics. Fig. 5 presents the original fingerprints together with experimental results. The exact same setup of the algorithm has been used on all fingerprints without any parameter tuning for any fingerprint image. The comparison between the proposed algorithm and a method described in [2], [3] is shown in Fig. 6.

\section{CONCLUSIONS}

The proposed adaptive fingerprint binarization algorithm shows a good ability to tune itself to each fingerprint image. This results in an algorithm which is insensitive to varieties in sensors, skin etcetera. Other than the initial setup, the algorithm is fully automatic.

\section{REFERENCES}

[1] D. Maltoni, D. Maio, A.K. Jain, and S. Prabhakar, Handbook of Fingerprint Recognition. Springer; 1st edition, 2003, ISBN 0-387-95431-7.

[2] L. O'Gorman and J.V. Nickerson, "Matched filter design for fingerprint image enhancement," in Proc. Int. Conf. on Acoustic Speech and Signal Processing, 1988, pp. 916-919. 


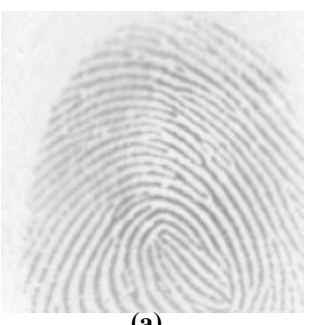

(a)
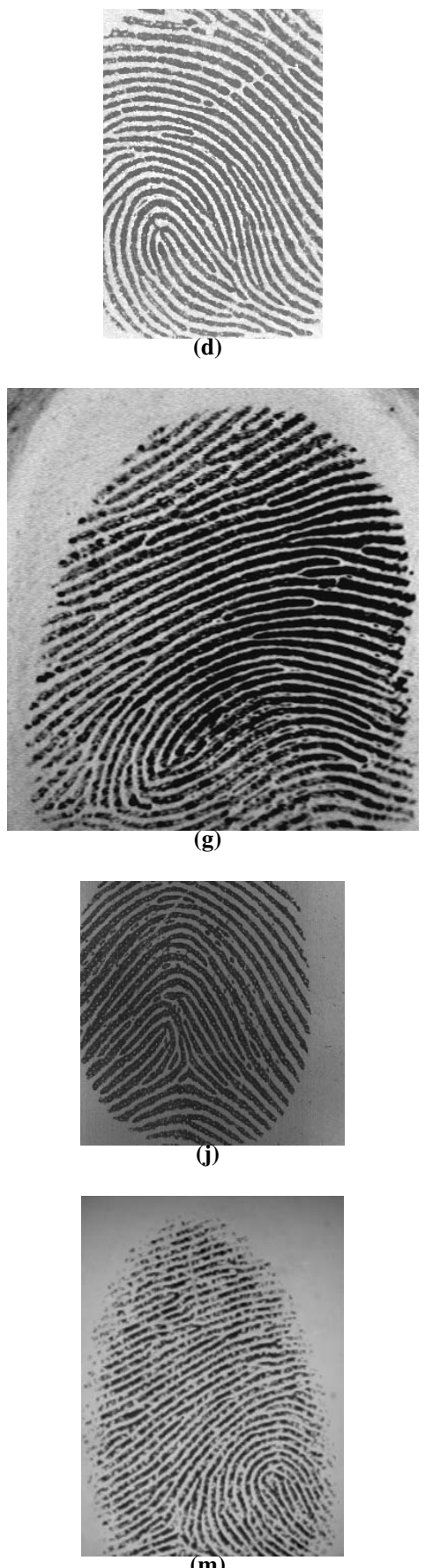

(m)
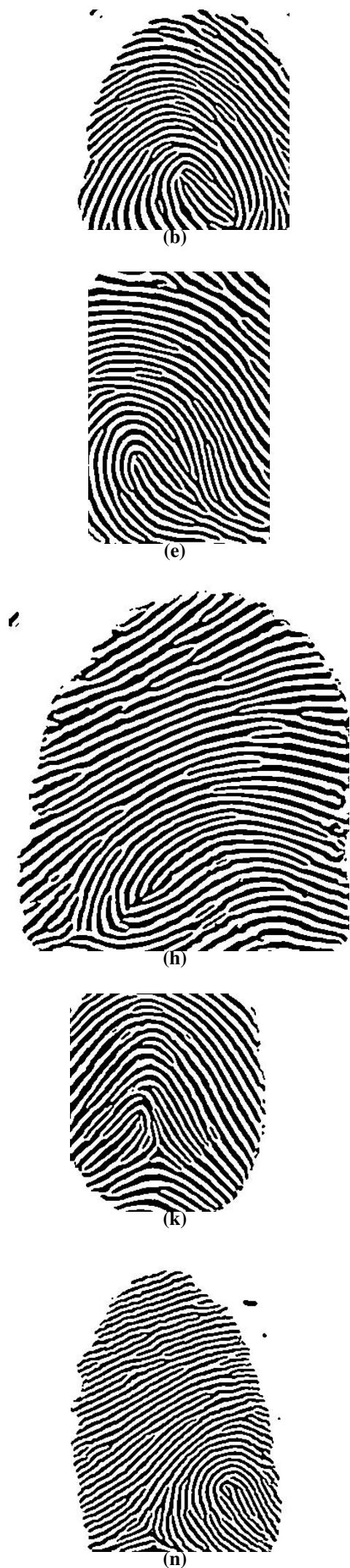

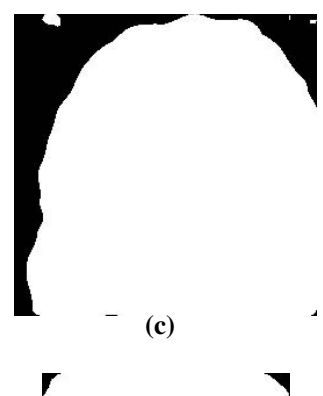

- (f)

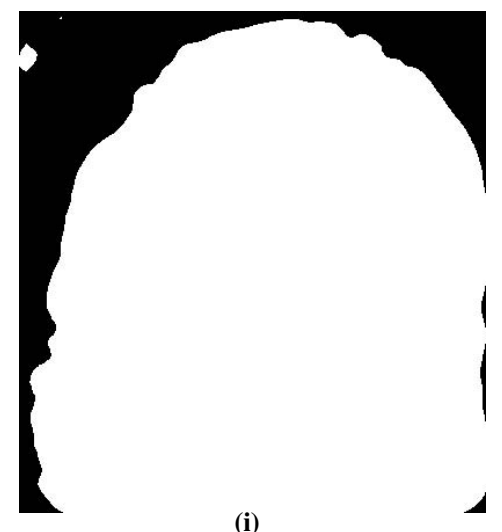

(i)
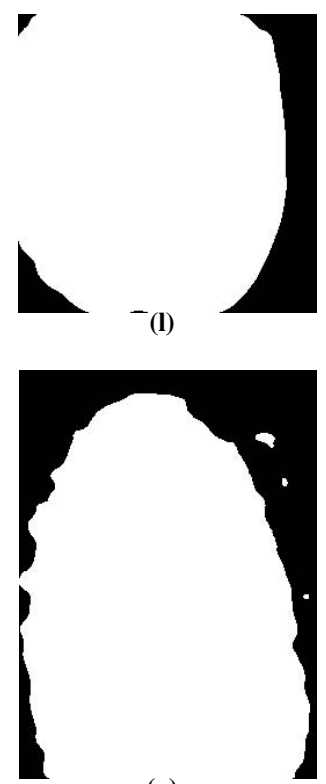

(o)

Fig. 5. Left column shows the gray-scale fingerprint images. Middle column shows the binarized fingerprint images generated with the proposed algorithm. Right column shows the mask of the fingerprints. The original image are obtained from databases in [1]. 


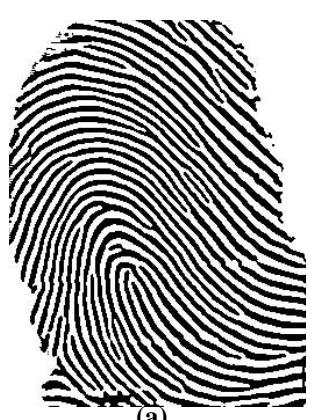

(a)

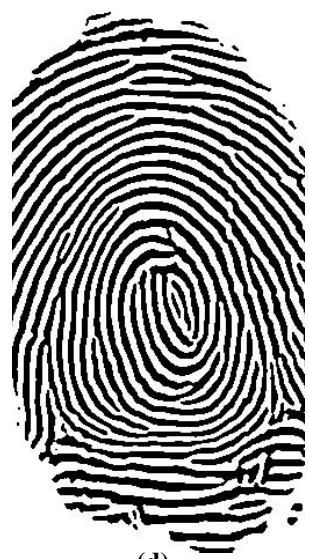

(d)
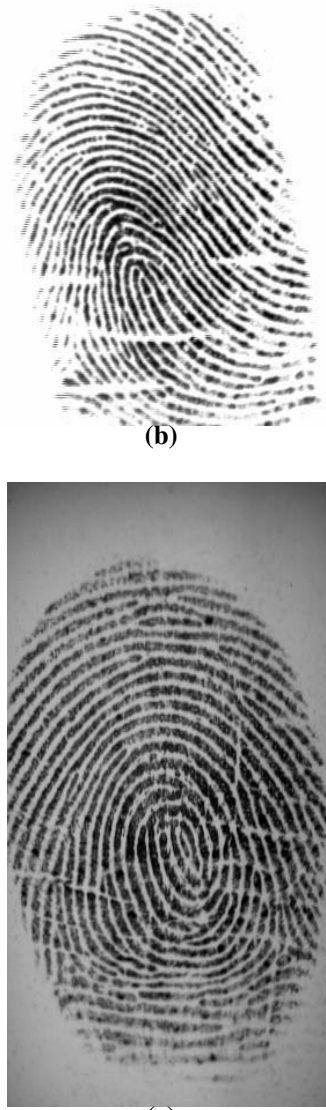

(e)
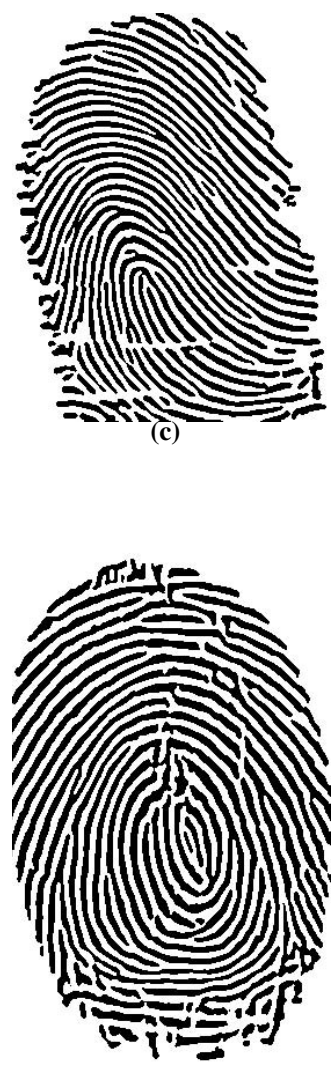

(f)

Fig. 6. Left column shows the binarized fingerprint images generated with the proposed algorithm. Middle column shows the gray-scale fingerprint images. Right column shows the binarized fingerprint images generated with method described in [2], [3].

[3] L. O’Gorman and J.V. Nickerson, "An approach to fingerprint filter design,” Pattern Recognition, vol. 22, no. 1, pp. 29-38, 1989.

[4] B.G. Sherlock, D.M. Monro, and K. Millard, "Fingerprint enhancement by directional fourier filtering," Vision, Image and Signal Processing, IEE Proceedings-, vol. 141, no. 2, pp. 87-94, 1994.

[5] L. Hong, Y. Wan, and A. Jain, "Fingerprint image enhancement: Algorithm and performance evaluation," IEEE Transactions on Pattern Analysis and Machine Intelligence, vol. 20, no. 8, pp. 777-790, 1998.
[6] M. Tico, M. Vehvilainen, and J. Saarinen, "A method of fingerprint image enhancement based on second directional derivatives," in IEEE International Conference on Acoustics, Speech, and Signal Processing (ICASSP), 2005, pp. 985-988.

[7] J. Lim, Two-Dimensional Signal and Image Processing. Prentice-Hall; 1st edition, 1990, ISBN 0-13-935322-4. 